\title{
Membrane damage as mechanism of photodynamic inactivation using Methylene blue and TMPyP in Escherichia coli and Staphylococcus aureus
}

\author{
Denise Muehler $^{1}$ (D) Elena Brandl ${ }^{2,3} \cdot$ Karl-Anton Hiller $^{1} \cdot$ Fabian Cieplik $^{1} \cdot$ Tim Maisch $^{3}$
}

Received: 24 June 2021 / Accepted: 11 December 2021 / Published online: 21 January 2022

(c) The Author(s) 2022

\begin{abstract}
The worldwide threat of antibiotic resistance requires alternative strategies to fight bacterial infections. A promising approach to support conventional antibiotic therapy is the antimicrobial photodynamic inactivation (aPDI). The aim of this work was to show further insights into the antimicrobial photodynamic principle using two photosensitizers (PS) of different chemical classes, Methylene Blue (MB) and TMPyP, and the organisms Escherichia coli and Staphylococcus aureus as Gramnegative and Gram-positive representatives. Planktonic cultures of both species were cultured under aerobic conditions for $24 \mathrm{~h}$ followed by treatment with MB or TMPyP at various concentrations for an incubation period of $10 \mathrm{~min}$ and subsequent irradiation for $10 \mathrm{~min}$. Ability to replicate was evaluated by CFU assay. Accumulation of PS was measured using a spectrophotometer. The cytoplasmic membrane integrity was investigated by flow cytometry using SYBR Green and propidium iodide. In experiments on the replication ability of bacteria after photodynamic treatment with TMPyP or MB, a killing rate of $5 \log _{10}$ steps of the bacteria was achieved. Concentration-dependent accumulation of both PS was shown by spectrophotometric measurements whereby a higher accumulation of TMPyP and less accumulation of MB was found for $S$. aureus as compared to E. coli. For the first time, a membrane-damaging effect of TMPyP and MB in both bacterial strains could be shown using flow cytometry analyses. Furthermore, we found that reduction of the replication ability occurs with lower concentrations than needed for membrane damage upon MB suggesting that membrane damage is not the only mechanism of aPDI using MB.
\end{abstract}

Keywords Antibacterial $\cdot$ Photodynamic $\cdot$ Methylene blue $\cdot$ TMPyP $\cdot$ Membrane damage $\cdot$ Flow cytometry

\section{Introduction}

Since the emergence of resistant bacteria shortly after the introduction of the first antibiotics, there has been a steady increase in antibiotic resistance worldwide [1]. Just

Denise Muehler and Elena Brandl contributed equally to this manuscript and share first authorship.

Denise Muehler

denise.muehler@ukr.de

1 Department of Conservative Dentistry and Periodontology, University Hospital Regensburg, Franz-Josef-Strauß-Allee 11, 93053 Regensburg, Germany

2 Institute of Microbiology and Archaea Centre, University of Regensburg, Regensburg, Germany

3 Department of Dermatology, University Hospital Regensburg, Regensburg, Germany less than one year after the development of the antibiotic methicillin in 1960, the first cases of the now well-known methicillin-resistant Staphylococcus aureus (MRSA) appeared [2]. In addition to antibiotic resistance, an adaptation of bacteria toward frequently used antiseptics has also become a subject of current research [3, 4]. In particular, multidrug-resistant bacterial strains such as Enterococcus spp., Staphylococcus aureus, Klebsiella pneumoniae, Acinetobacter baumannii, Pseudomonas aeruginosa and Enterobacter spp., also known as ESKAPE pathogens and most commonly associated with nosocomial infections, have become a severe problem for public healthcare [5, $6]$. It is estimated that by 2050 , there will be more deaths associated with antimicrobial resistance than with cancer, amounting to ten million deaths per year [7]. In addition to measures such as prudent use of antibiotics, improved hygiene measures and international cooperation, it is urgent to develop new alternative antibacterial strategies 
to combat infections caused by resistant bacterial pathogens $[8,9]$.

Antimicrobial photodynamic inactivation (aPDI) represents a promising method for inactivating bacteria $[8$, 10-12]. This process generates reactive oxygen species (ROS) that cause non-specific and irreversible oxidative damage to biological molecules, which results in the killing of bacteria. A combination of three components is essential for the mechanism of action of photodynamic inactivation: a non-toxic dye, the so-called photosensitizer (PS), light of appropriate wavelength to excite the PS, and molecular oxygen $\left(\mathrm{O}_{2}\right)$. Two different mechanisms are involved in this process: type I mechanism where electron transfer to oxygen causes the formation of superoxide $\left(\mathrm{O}_{2}^{-\cdot}\right)$ and eventually production of cytotoxic hydroxyl radicals ( $\mathrm{HO}^{*}$ ), and type II mechanism involving the transfer of energy to ground-state triplet oxygen causing the production of singlet oxygen $\left({ }^{1} \mathrm{O}_{2}\right)$. The relative amount of type II mechanism is described by the singlet oxygen quantum yield $\Phi_{\Delta}[9,13,14]$. ROS formed in both mechanisms lead to damage of biological molecules such as lipids, proteins, enzymes, DNA and RNA. The actual oxidative damage caused by aPDI depends on the localization of the photosensitizer to these structures and on the time-limited diffusion length of the resulting ROS $[9,13]$.

Different classes of PS have been discovered and explored in recent years [8]. Methylene blue (MB), belonging to the class of phenothiazinium dyes, is used for aPDI, e.g., in dental practice [15-17]. This photosensitizer acts by type I mechanism, but also by type II mechanism with $\Phi_{\Delta}=0.52[18,19]$. Advantages of MB are its positive charge, low dark toxicity and the ability to penetrate or attach to biological membranes. Previous studies have shown its high efficacy toward different microorganisms e.g., E. coli [20], S. aureus [21] or Candida albicans [22]. Another PS frequently employed for aPDI is TMPyP, which is based on a porphyrin structure. The fourfold positively charged derivate predominantly acts by type II mechanism upon light irradiation with $\Phi_{\Delta}=0.74$ achieving high inactivation efficacy of different pathogens in vitro [18, 23-26].

While the antibacterial efficacy of both PS toward planktonic bacteria is well established by many studies, little is known about the exact mechanism that leads to the inactivation of bacterial cells [8]. Therefore, the aim of the present study was to investigate the mechanism of action of aPDI using MB and TMPyP as PS toward planktonic cultures of Gram-negative E. coli or Gram-positive $S$. aureus by combining different methodologies. For this purpose, a colony-forming units (CFU) assay was used to investigate reductions of bacterial ability to replicate following treatment. Accumulation of the PS by the bacterial cells was measured spectrophotometrically, and damage of bacterial cytoplasmic membranes following treatment was investigated by flow cytometry.

\section{Material and methods}

\subsection{Chemicals and light sources}

MB [(7-(dimethylamino) phenothiazin-3-ylidene)-dimethylazanium; chloride] and TMPyP (5,10,15,20-tetrakis(1methylpyridinium-4-yl)-porphyrin tetrap-toluenesulfonate) were purchased from Sigma Aldrich (St. Louis, MO, USA). Two-fold dilutions from 0 to $31.25 \mu \mathrm{M}$ were prepared from a $1 \mathrm{mM}$ stock solution with demineralized ultrapure water $\left(\mathrm{H}_{2} \mathrm{O}\right)$ (Milli Q Biocel, Merck KGaA, Darmstadt, Germany) for both PS. PS-solutions were stored at $4{ }^{\circ} \mathrm{C}$ for no longer than 2 weeks. Chlorhexidine digluconate solution (CHX) was obtained from Sigma Aldrich in a concentration of $20 \%$ and was further dissolved in $\mathrm{H}_{2} \mathrm{O}$. For irradiation of $\mathrm{MB}$, the light source PDT 1200L (Herbert Waldmann GmbH \& Co. KG, VillingenSchwenningen, Germany) exhibiting an irradiance of 20 $\mathrm{mW} / \mathrm{cm}^{2}$ was used. TMPyP was irradiated with the light source UV 236 (Herbert Waldmann GmbH \& Co. KG) obtaining an output of $18 \mathrm{~mW} / \mathrm{cm}^{2}$. Both PS were irradiated for $10 \mathrm{~min}$, resulting in light doses of $12 \mathrm{~J} / \mathrm{cm}^{2}$ for MB and $10.8 \mathrm{~J} / \mathrm{cm}^{2}$ for TMPyP.

\subsection{Bacterial culture}

E. coli (ATCC 25922) and S. aureus (ATCC 25923) were obtained from the German Collection of Microorganisms and Cell Cultures (DSMZ; Braunschweig, Germany) to be used in this study. The bacterial strains were stored at $-80^{\circ} \mathrm{C}$. Bacteria were cultured under aerobic conditions in Mueller-Hinton (MH) broth (Merck KgaA, Darmstadt, Germany) or on MH agar plates (Institute of Microbiology and Hygiene, University Hospital Regensburg, Germany). For the preparation of planktonic cultures, single colonies were picked and cultured in $5 \mathrm{~mL}$ MH broth over-night at $37^{\circ} \mathrm{C}$ at $160 \mathrm{rpm}$ on an orbital shaker. Optical density (OD) of a bacterial suspension was determined by measuring the absorbance at $600 \mathrm{~nm}$ wavelength $\left(\mathrm{OD}_{600}\right)$ in a spectrophotometer (SPECORD ${ }^{\circledR} 50$ PLUS, Analytik Jena, Jena, Germany). Over-night cultures were harvested by centrifugation (5000 $g ; 10 \mathrm{~min}$ ), and bacterial cell pellets were resuspended in $5 \mathrm{~mL}$ PBS (Dulbecco's phosphate-buffered saline; Gibco, Thermo Fisher Scientific, Waltham, MA, USA). Bacteria were diluted in PBS to yield an $\mathrm{OD}_{600}$ of 0.6 (CFU experiments) or 1.0 (flow cytometry and accumulation experiments), respectively. 


\subsection{Assessment of bacterial ability to replicate by CFU assay}

$100 \mu \mathrm{L}$ PS solution was mixed with $100 \mu \mathrm{L}$ of the bacterial suspension $\left(\mathrm{OD}_{600} 0.6\right)$ in a 96 -well plate (Ref. 3598, Corning Inc., Corning, NY, USA). Samples were incubated for $10 \mathrm{~min}$ at room temperature in the dark. Then, irradiation was performed for $10 \mathrm{~min}$. For each irradiated sample, a corresponding dark control was incubated in the dark for $20 \mathrm{~min}$. An unirradiated negative control and an irradiated positive control each treated with $\mathrm{H}_{2} \mathrm{O}$ instead of a PS were included in each experiment. Ten-fold serial dilutions $\left(10^{-3}-10^{-8}\right)$ were prepared in PBS and aliquots (20 $\mu \mathrm{L}$ ) were plated on $\mathrm{MH}$ agar plates according to the method described by Miles et al. [27]. Afterwards, agar plates were incubated aerobically for $24 \mathrm{~h}$. Subsequently, CFUs were evaluated. Three independent experiments were performed in duplicates.

\subsection{PS accumulation measurements}

Equal amounts $(500 \mu \mathrm{L})$ of the bacterial suspensions $\left(\mathrm{OD}_{600}\right.$ $1.0)$ and the corresponding PS-solution were mixed. After 10 min incubation in the dark, the samples were centrifuged $(24 \times 3,75 \mathrm{~g} ; 10 \mathrm{~min})$ and then the supernatants were further used to determine non-attached PS. The transmission spectra of such supernatants were measured with a spectrophotometer (SPECORD ${ }^{\circledR} 50$ PLUS) in a wavelength range from 380 to $800 \mathrm{~nm}$. Additionally, control measurements were performed of bacterial suspensions without the respective PS. Spectral reference measurements of solutions containing different concentrations of TMPyP (from 0 to $3.90 \mu \mathrm{M}$ ) or MB (from 0 to $15.63 \mu \mathrm{M}$ ) only were done as well but without bacteria. Each transmission spectra were converted into the respective absorption spectra by Lambert-Beer law (100\% transmission corresponds to 0\% absorption). Finally, the absorption maxima of each probe were subtracted from the corresponding reference measurement. Three independent experiments were performed.

\subsection{Flow cytometric analysis of membrane integrity}

Flow cytometry was used to examine bacterial membrane integrity. Propidium iodide (PI; Sigma-Aldrich, St. Louis, MO, USA) and SYBR Green I (SG; Thermo Fisher Scientific, Waltham, MA, USA) were used as fluorescent dyes to evaluate the integrity of cytoplasmic membranes. Bacterial samples were cultured and treated as described above. All centrifugation steps took place at $24 \times 3,75 \mathrm{~g}$ for $10 \mathrm{~min}$ and $4{ }^{\circ} \mathrm{C}$. As a positive control for membrane damage, bacteria were treated with $0.1 \%$ chlorhexidine digluconate (CHX) for $20 \mathrm{~min}$ (RT) as described [10]. After treatment, cells were centrifuged, washed with $1 \mathrm{~mL}$ PBS, and resuspended in $1 \mathrm{~mL}$ PBS. For E. coli, $942 \mu \mathrm{L}$ PBS with $50 \mu \mathrm{L}$ bacterial suspension were stained with $1 \mu \mathrm{L} \mathrm{SG}(1: 10)$ for $15 \mathrm{~min}$ and then with $7 \mu \mathrm{L}$ PI $(7 \mu \mathrm{g} / \mathrm{ml})$ for $5 \mathrm{~min}$. For $S$. aureus, 496 $\mu \mathrm{L}$ bacterial suspension were directly stained with $1 \mu \mathrm{L}$ SG (1:200) and $3 \mu \mathrm{L}$ PI $(3 \mu \mathrm{g} / \mathrm{ml})$ the same way. Different quantities of bacterial cell suspension were used to reach a flow rate of 1000 cells/s. Furthermore, different concentrations of SG and PI were used due to the difference in dye uptake by both species. After incubation, samples were mixed and measured with a BD FACSCanto II flow cytometer (Becton Dickinson, Franklin Lakes, NJ, United States) equipped with a $488 \mathrm{~nm}$ air-cooled solid-state laser with the output of $20 \mathrm{~mW}$. PI fluorescence (FL3) was analyzed using a 650 long-pass filter, SG fluorescence (FL1) was analyzed by a $530 / 30$ bandpass filter. Bacterial cells were gated on FSC/ SSC dot plots from which FL3/FL1 dot plots were derived. A total of 10,000 events were evaluated. Data acquisition was performed with the FACSDiva ${ }^{\mathrm{TM}}$ 5.0.2 software (Becton Dickinson) and data were analyzed using the Flow $\mathrm{Jo}^{\circledR}$ software, version 10 (FlowJo, LLC, Ashland, OR, USA). From all fluorescence signals, the background signal was subtracted. Three independent experiments were performed.

\subsection{Data analysis}

CFU results are shown as medians, 1st and 3rd quartiles. Horizontal solid and dashed lines in the graphs represent reductions of $3 \log _{10}$ and $5 \log _{10}$ steps of CFU, respectively, compared to the untreated control group UC L-. Medians on or below these lines demonstrate an antimicrobial efficacy of $99.9 \%\left(3 \log _{10}\right)$ or $99.999 \%\left(5 \log _{10}\right)$, at least, which is declared as biologically relevant antimicrobial activity (3 $\left.\log _{10}\right)$ or disinfectant effect $\left(5 \log _{10}\right)$ according to guidelines of infection control [28, 29]. For accumulation experiments, the absorption maxima (recorded as \%) of the supernatant (PS solution and bacteria) were subtracted from the median of the reference measurement (PS solution without bacteria) to obtain the absorption difference, expressed as uptake (\%). Values from uptake and flow cytometry experiments were presented as median, minimum, and maximum. All calculations were performed using SPSS, v. 25 (SPSS Inc., Chicago, IL, USA). Statistical evaluation was performed using the Tukey method.

\section{Results}

\subsection{Assessment of bacterial ability to replicate by CFU assay}

The bacterial ability to replicate following aPDI with MB and TMPyP was evaluated by CFU assay against planktonic cultures of E. coli and S. aureus (Fig. 1). Treatment 
A E. coli-Methylene blue

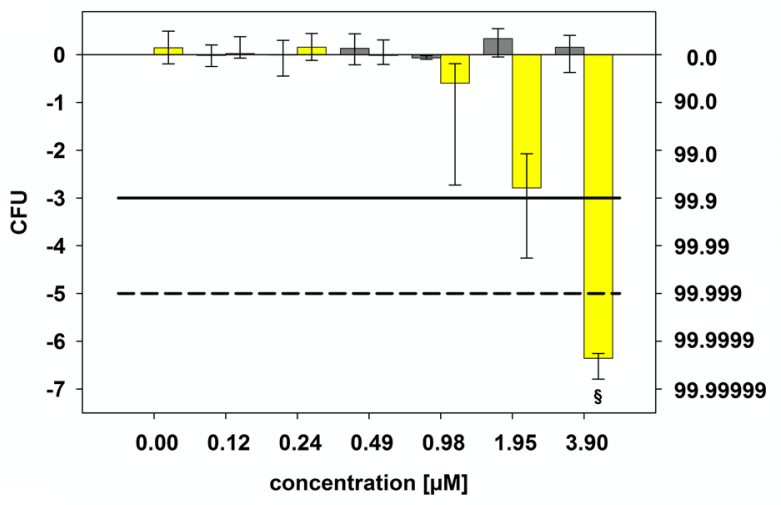

C

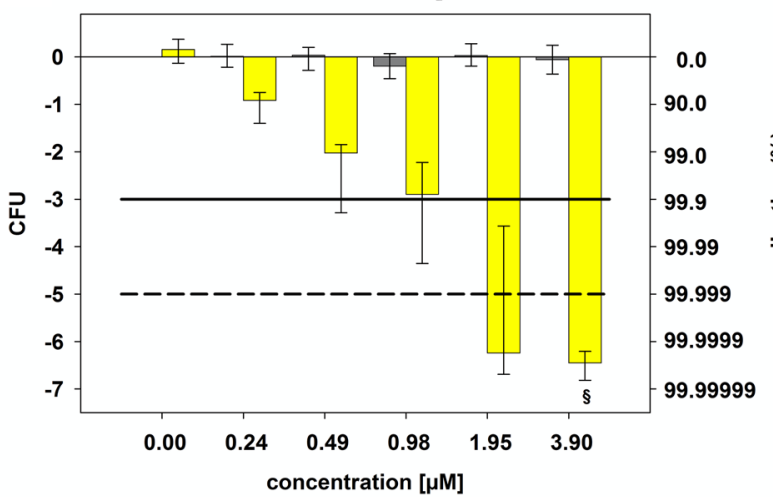

Fig. 1 Antimicrobial efficacy of PDI with MB and TMPyP toward $E$. coli and $S$. aureus. All results are depicted as medians, 1st and 3rd quartiles on a $\log _{10}$-scaled ordinate. Gray bars represent dark groups; yellow bars represent irradiated groups. Horizontal solid and dashed lines represent CFU-reductions of $3 \log _{10}$ and $5 \log _{10}$ steps, respec-

with light or PS alone had no effect in all cases. Treatment with aPDI with MB or TMPyP resulted in concentrationdependent reductions of $\mathrm{CFU}$ in both bacterial species. For aPDI with MB $(3.9 \mu \mathrm{M})$ and TMPyP $(3.9 \mu \mathrm{M})$, there were CFU reductions of up to $6.4 \log _{10}$ and $6.5 \log _{10}$ steps for $E$. coli and up to $6.3 \log _{10}$ and $6.7 \log _{10}$ steps for $S$. aureus, respectively.

\subsection{PS accumulation measurements}

The amount of accumulation was determined spectrophotometrically for MB and TMPyP by measuring the absorption spectra of the respective PS remaining in the supernatants of planktonic cultures which were incubated with the respective PS for $10 \mathrm{~min}$. The absorbance difference (uptake (\%)) of the absorption spectra of the supernatant containing PS (standard concentration row) but not bacteria vs. supernatant of bacterial suspension incubated with the respective PS, represents the theoretically accumulated or absorbed amount of PS onto bacteria (Fig. 2A and B). The absorbance

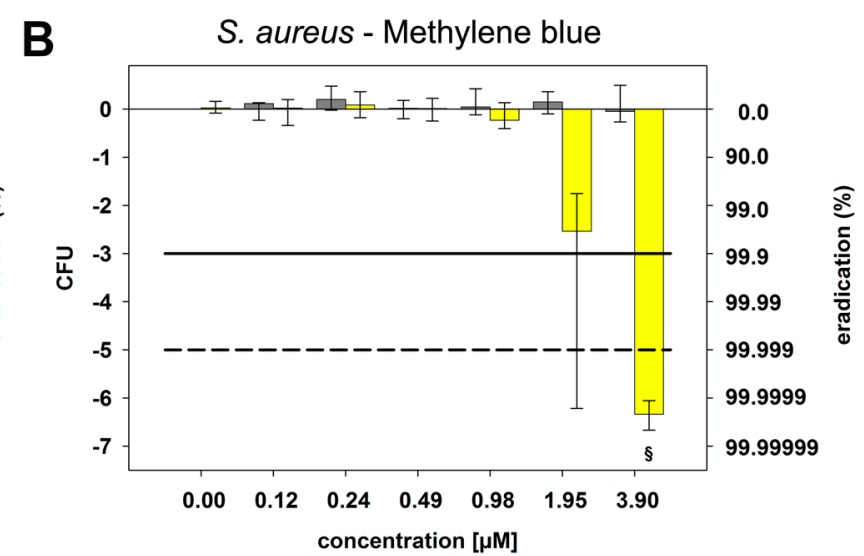

D

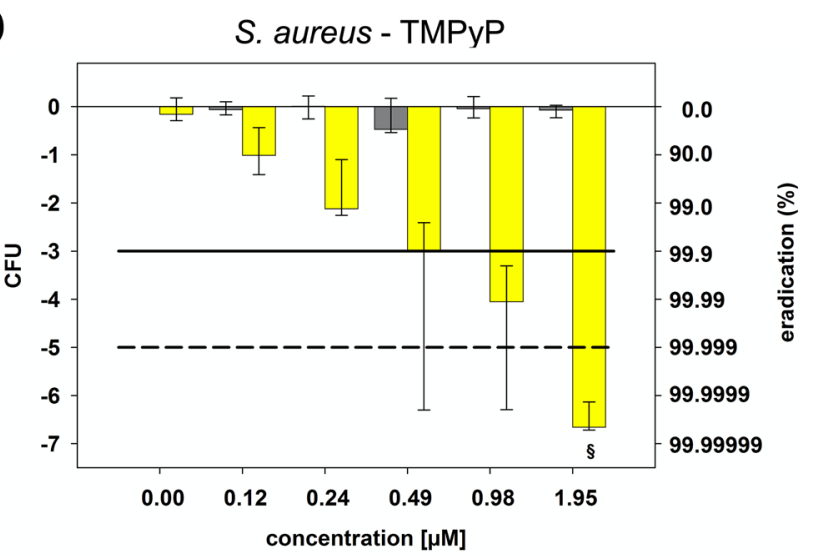

tively, related to untreated $(0 \mu \mathrm{M}$, non-irradiated $)$ controls. Bacterial cells were incubated with each PS for $10 \mathrm{~min}$ and irradiated for another $10 \mathrm{~min}$. A E. coli upon treatment with $\mathrm{MB}$; B $S$. aureus upon treatment with MB; C E. coli upon treatment with TMPyP; D $S$. aureus upon treatment with TMPyP; $\$$ : below detection limit; $n=6$

difference values of the supernatants represent the theoretically accumulated or absorbed photosensitizer amounts in percent (Fig. 2A and B). For both, E. coli and S. aureus, a concentration-dependent accumulation of MB and TMPyP was detected based on the tested concentration range. This is shown by the absorbance difference of the supernatant $>0 \%$ (Fig. 2A and B).

\subsection{Flow cytometric analysis of membrane integrity}

To evaluate membrane integrity, flow cytometry with PI and SG as fluorescent dyes was employed upon aPDI treatment with MB and TMPyP. Figure 3 shows the region of interest (ROI) determined in untreated controls for both bacterial species on dot plots FSC vs. SSC (Fig. 3A and D), dot plots of untreated bacterial cells (Fig. 3B and E) and bacterial cells treated with $0.1 \% \mathrm{CHX}$ as a positive control for a membrane damaging agent (Fig. 3C and F). Figures 4 and 5 show exemplary dot plots of untreated bacterial cells (Figs. 4A, D, $5 \mathrm{~A}$ and $\mathrm{D}$ ) and bacterial cells treated with aPDI using MB 


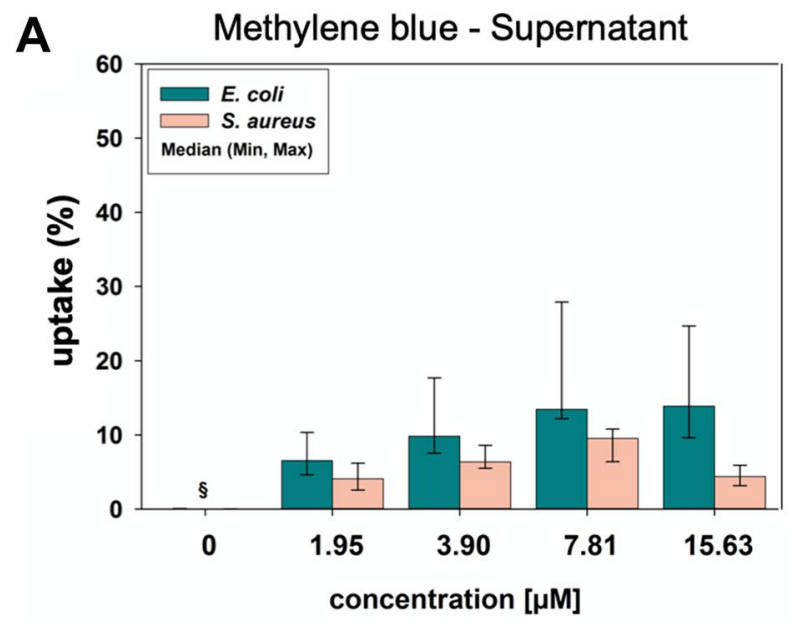

Fig. 2 Accumulation measurements with $\mathrm{MB}$ and TMPyP for $E$. coli and $S$. aureus. Difference of the absorption maxima in percent (uptake (\%)) for $E$. coli and $S$. aureus treated each with MB or TMPyP. Values of supernatant are represented as medians with
B

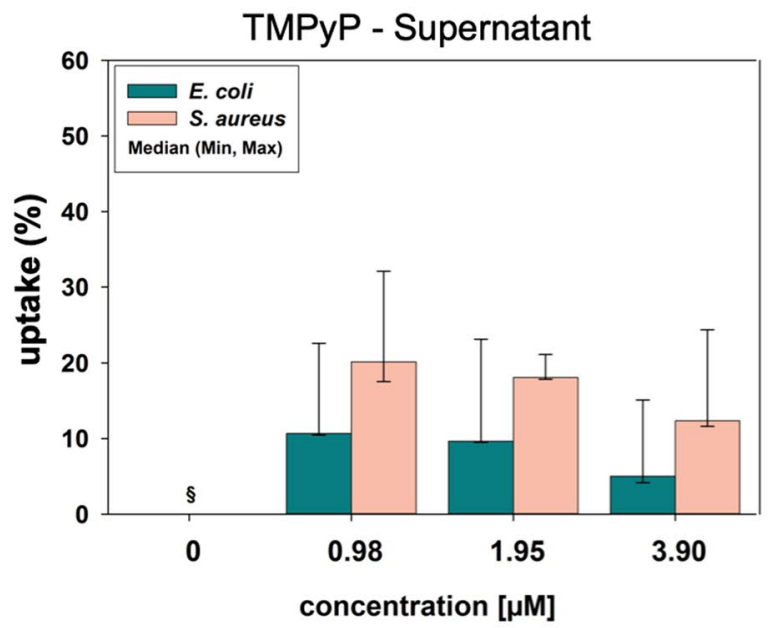

minima and maxima. A Absorption differences of supernatants upon treatment with $\mathrm{MB} ; \mathbf{B}$ absorption differences of supernatants upon treatment with TMPyP; D Negative control without PS $=0 ; \xi=$ value measured; $n=3$
A

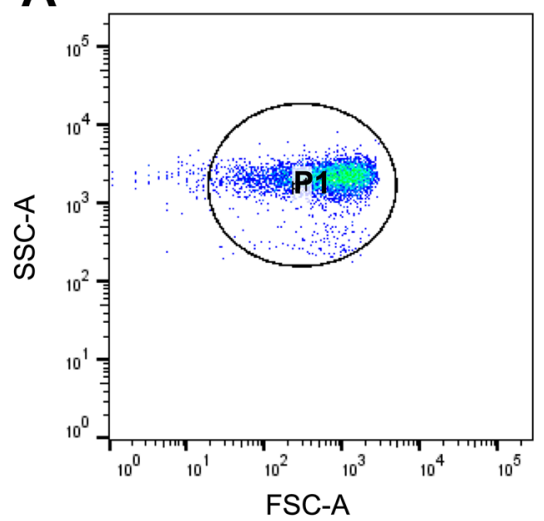

D

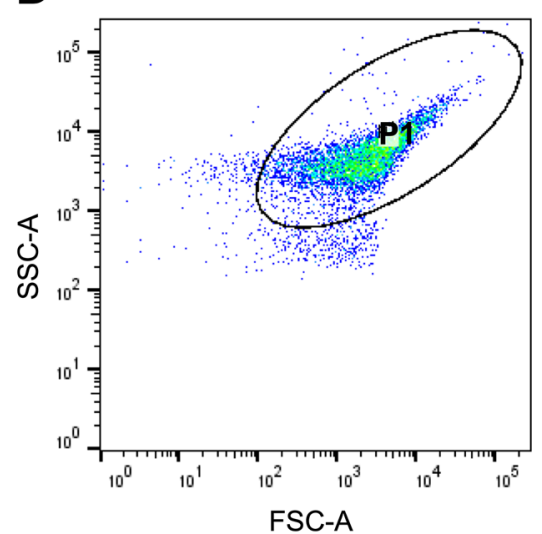

B

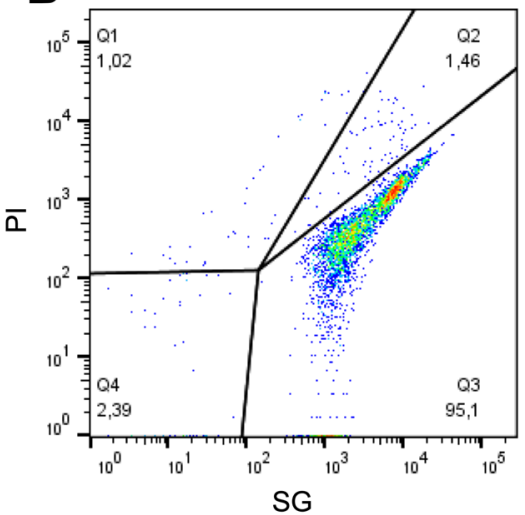

E

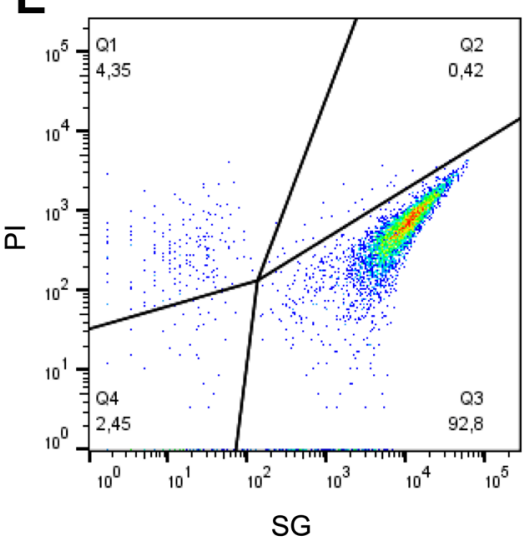

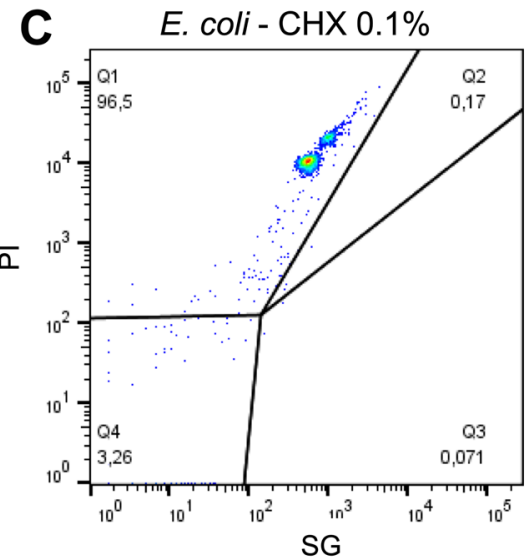

$\mathbf{F}$

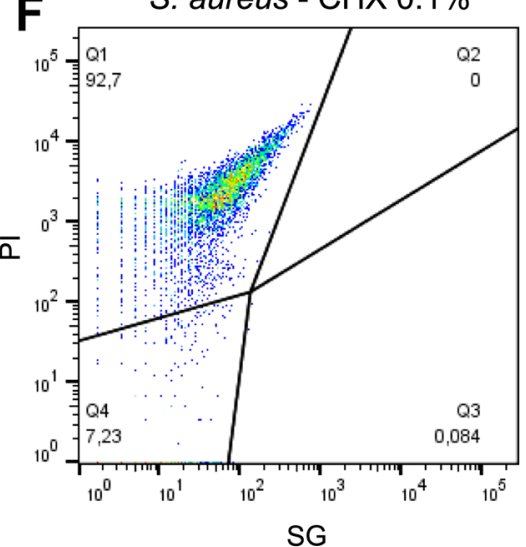

Fig. 3 Flow cytometry data for membrane integrity upon treatment with $0.1 \% \mathrm{CHX}$ as a positive control. Bacterial cell populations gated on dot plot FSC-A vs. SSC-A showing the chosen region of interest (ROI) for A E. coli and D S. aureus. Exemplary logarithmic dot plots of fluorescence intensity for SYBR Green (SG) and propidium iodide (PI) of $\mathbf{C}$ untreated $E$. coli and $\mathbf{F}$ untreated $S$. aureus and for $\mathbf{C}$ E. coli and $\mathbf{F}$ S. aureus after treatment with $0.1 \%$ CHX. Distribution of bacterial cell population in percent for each quadrant: Q1 (PI), Q2 (SG+PI), Q3 (SG) and Q4 (unstained) 


\section{E. coli}

A

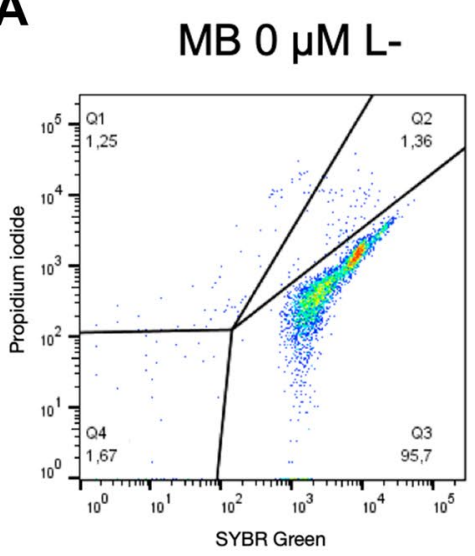

D

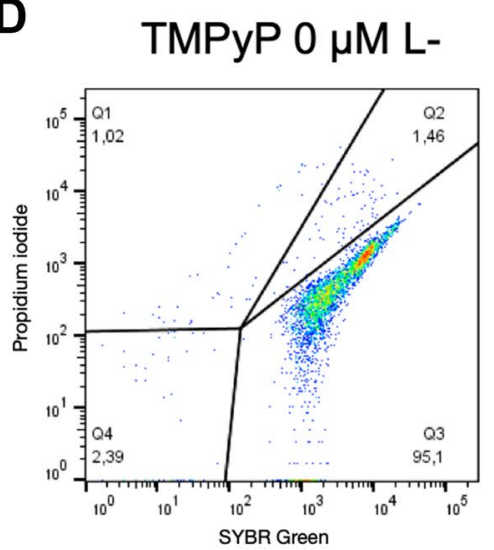

B

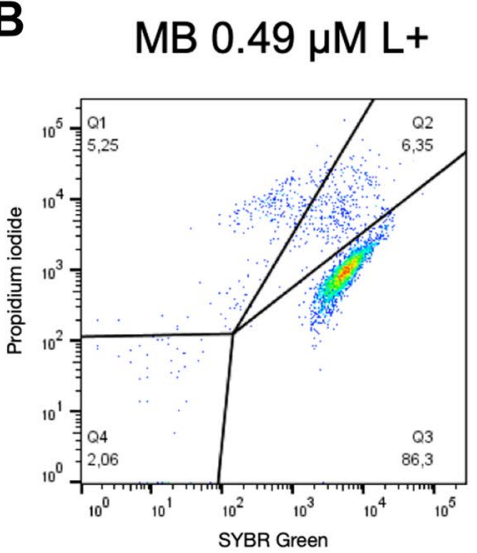

E TMPyP $0.24 \mu \mathrm{M} \mathrm{L+}$

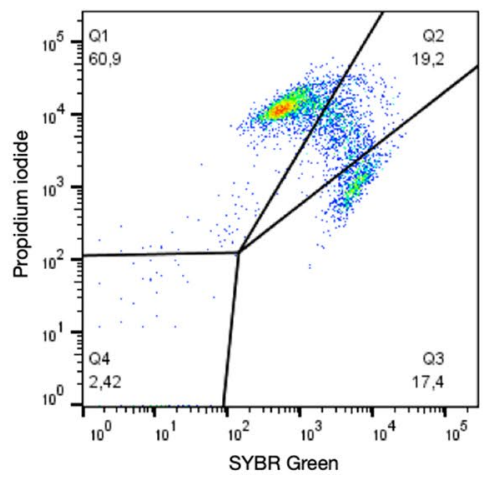

C $\quad \mathrm{MB} 3.9 \mu \mathrm{ML}+$

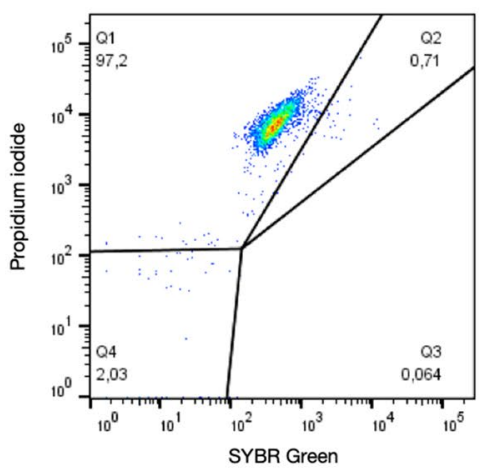

F TMPyP $1.95 \mu \mathrm{ML+}$

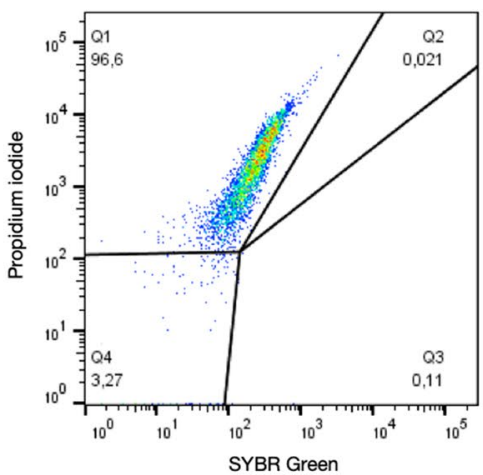

Fig. 4 Flow cytometry data for membrane integrity in E. coli upon treatment with aPDI using methylene blue (MB) or TMPyP. Exemplary logarithmic dot plots of fluorescence intensity for SYBR Green (SG) and propidium iodide of $\mathbf{A}$ and $\mathbf{D}$ untreated E. coli, B E. coli treated with $0.49 \mu \mathrm{M} \mathrm{MB}, \mathbf{C}$ treated with $3.9 \mu \mathrm{M} \mathrm{MB}, \mathbf{E}$ treated with
$0.24 \mu \mathrm{M}$ TMPyP and F treated with $1.95 \mu \mathrm{M}$ TMPyP. Distribution of bacterial cell population in percent for each quadrant: Q1 (PI), Q2 (SG + PI), Q3 (SG) and Q4 (unstained). L-: not irradiated, L+ : irradiated

\section{Discussion}

Numerous in vitro studies have clearly demonstrated the antibacterial efficacy of PDI [8-10, 30]. However, the exact antibacterial mechanism of the photodynamic process has not yet been completely understood. This is of great importance to estimate possible developments of tolerances and resistances and also to be able to improve the effectiveness of the photosensitizers by chemical modifications. Thus, in this work, we focused on the investigation of the mechanism of action of the well-studied PS MB and TMPyP.

For this purpose, the ability to replicate was examined after treatment with different concentrations of $\mathrm{MB}$ and TMPyP. Compared to Gram-negative E. coli, for Grampositive $S$. aureus lower concentrations of TMPyP and approximately equal concentrations of $\mathrm{MB}$ were required for an effective reduction of CFU by $3 \log _{10}$ or $5 \log _{10}$ steps, 


\section{S. aureus}

A

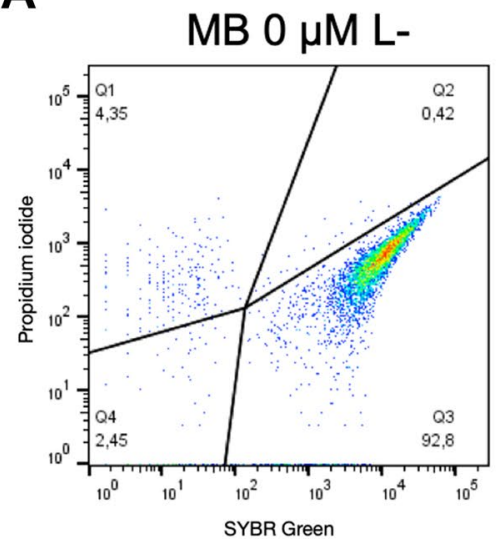

D

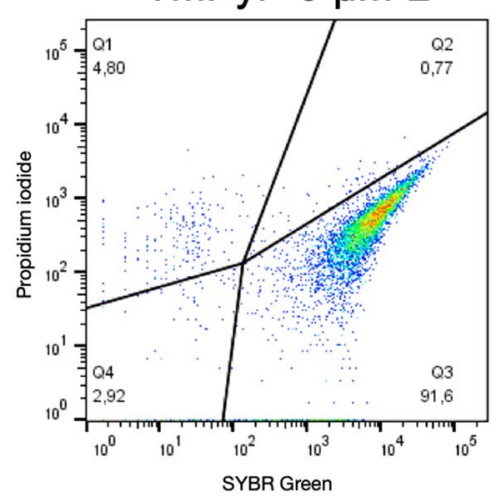

B

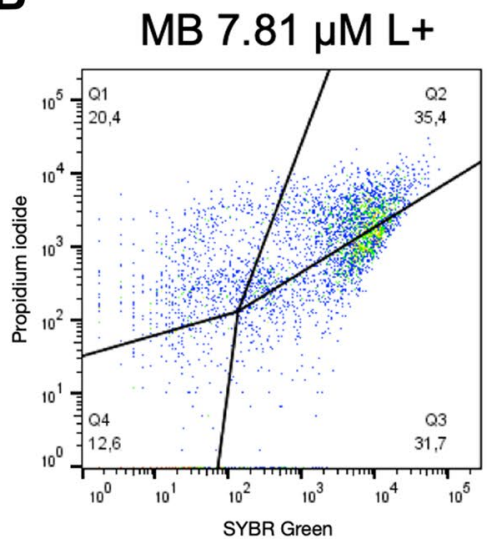

E
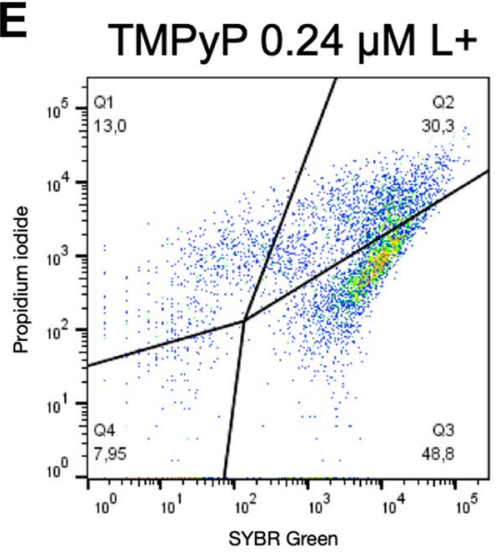

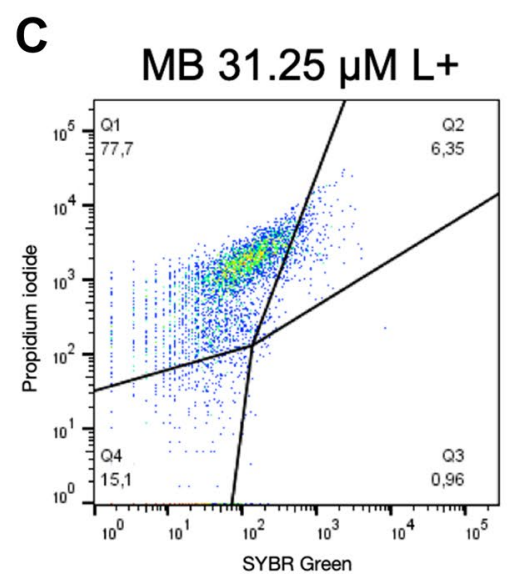

$F$

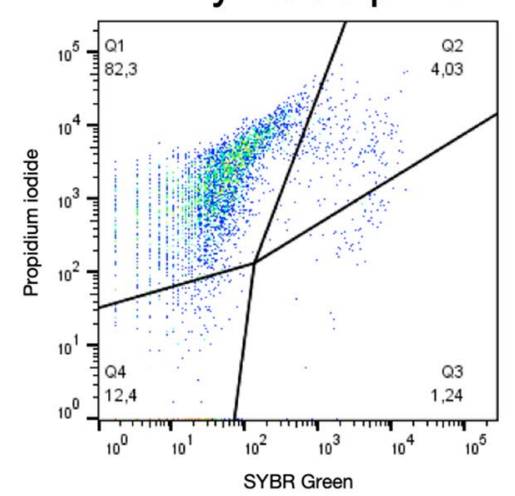

Fig. 5 Flow cytometry data for membrane integrity in $S$. aureus upon treatment with aPDI using methylene blue (MB) or TMPyP. Exemplary logarithmic dot plots of fluorescence intensity for SYBR Green (SG) and propidium iodide of $\mathbf{A}$ and $\mathbf{D}$ untreated $S$. aureus, B S. aureus treated with $0.49 \mu \mathrm{M} \mathrm{MB}, \mathbf{C}$ treated with $3.9 \mu \mathrm{M} \mathrm{MB}, \mathbf{E}$

respectively. Due to their different cell wall structure, Grampositive bacteria are more susceptible toward photodamage than Gram-negative bacteria $[8,31,32]$. The antibacterial efficacy of both PS as measured by CFU assay in this work is in accordance with other studies investigating the efficacy of aPDI using MB and TMPyP as PS [25, 26, 33-35]. In general, for both species, treatment with TMPyP showed higher reductions of CFU than treatment with MB. This might be due to the fourfold positive charges of TMPyP which enhances the attachment of the molecule to negatively charged bacterial membranes [36]. Furthermore, since ${ }^{1} \mathrm{O}_{2}$ is known to be the predominant ROS in the mechanism of aPDI, the higher killing efficacy of TMPyP may also be due to its higher ${ }^{1} \mathrm{O}_{2}$ quantum yield as compared to $\mathrm{MB}(0.74$ vs. 0.52).

As one PS molecule is excited by the absorption of one photon, adjusting the numbers of absorbed photons of treated with $0.24 \mu \mathrm{M}$ TMPyP and $\mathbf{F}$ treated with $1.95 \mu \mathrm{M}$ TMPyP. Distribution of bacterial cell population in percent for each quadrant: Q1 (PI), Q2 (SG + PI), Q3 (SG) and Q4 (unstained). L-: not irradiated, $\mathrm{L}+$ : irradiated

distinct PS-light source systems allows proper comparison of the antimicrobial photodynamic efficacy of different PS that are activated by different light sources [37]. Therefore, the irradiation parameters were adjusted for the two PS accordingly by applying a formula presented by Cieplik et al.: For each single wavelength, the energy of a single photon, the spectral radiant power of the light source and the absorption of the PS goes into the calculation of the formula. The sum is calculated for wavelengths ranging from 300 to $800 \mathrm{~nm}$ in 1-nm-steps. Therefore, the whole overlap of the absorption spectrum of the PS and the emission spectrum of the light source is considered [37]. Here, both PS-light source systems were adjusted to a number of $10^{16}$ photons that were absorbed during the irradiation period. Due to this adjustment based on numbers of absorbed photons, it can be concluded that TMPyP exhibited an overall higher antimicrobial photodynamic efficacy than MB under the given conditions. 

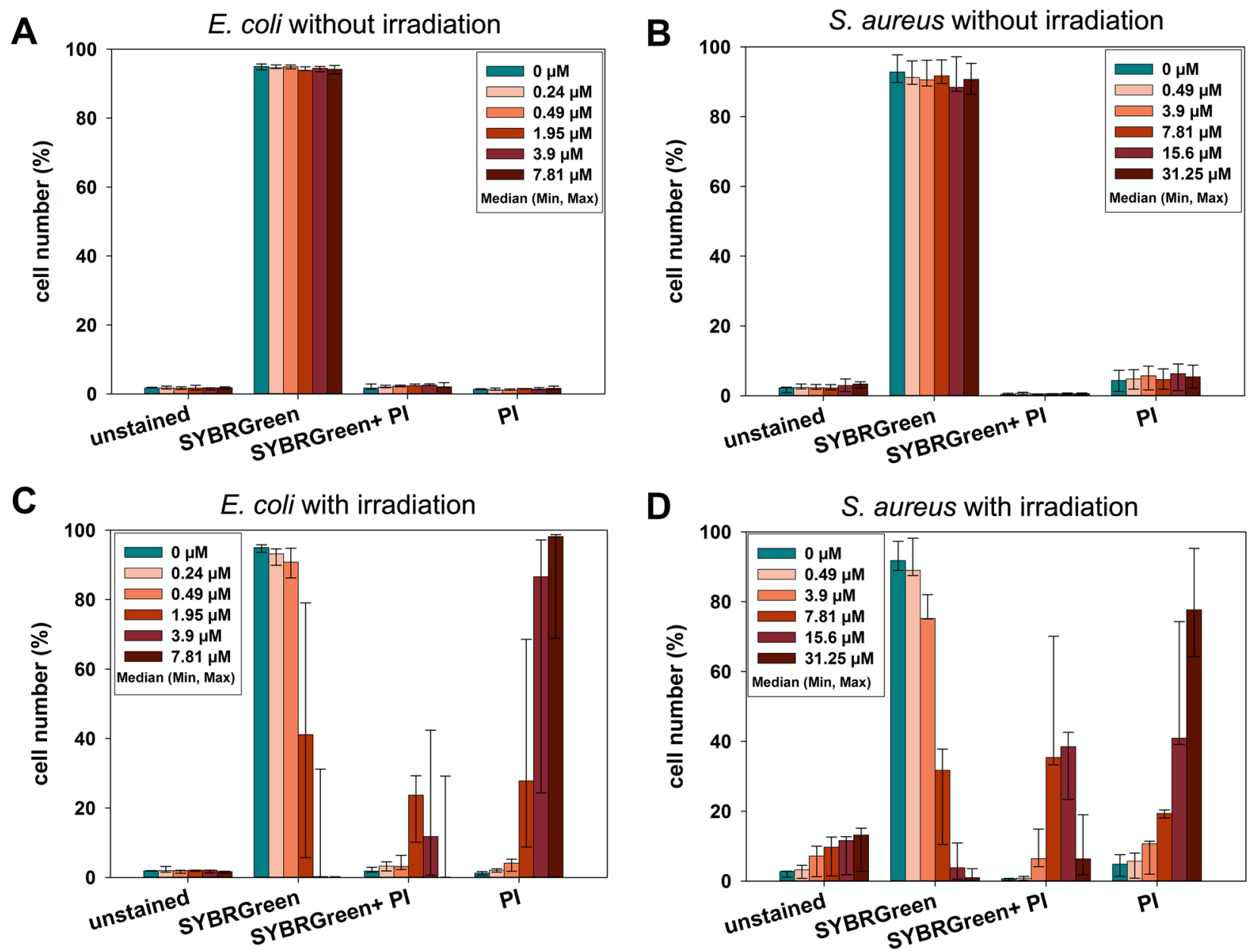

Fig. 6 Flow cytometry data on membrane integrity upon treatment of $E$. coli and $S$. aureus with MB. Bacterial cell number in percent for unstained, SG-stained, SG+PI-stained, or PI-stained cells are shown. Values are presented as median, minimum, and maximum. A Values in percent for $E$. coli upon treatment with MB without irradiation; B

The localization of the PS on or inside the bacterial cell is of great importance for the photodynamic process [38]. Therefore, we investigated the accumulation of both PS on the bacterial membranes spectrophotometrically. In accordance with previous studies, it was shown for both strains, E. coli and S. aureus, that TMPyP and MB are attached or taken up [39, 40]. Faraj Tabrizi et al. also showed uptake of TMPyP and MB in E. coli-K12 [40]. Another study demonstrated uptake or attachment of MB in S. aureus and E. coli via photometric measurements [39]. In the present work, a higher accumulation of TMPyP and less accumulation of MB was found for $S$. aureus as compared to $E$. coli. These differences may be due to the different charge distribution on the cell surfaces of Gram-positive and Gram-negative bacteria. Sonohara et al. revealed that E. coli has a more negatively charged surface than $S$. aureus [41]. Accordingly, the different number of positive charges of both PS may also

Values in percent for $S$. aureus upon treatment with MB without irradiation; C Values in percent for $E$. coli upon treatment with MB with irradiation; D Values in percent for $S$. aureus upon treatment with MB with irradiation; $n=3$

play an important role in the accumulation of the PS, as already discussed above for antibacterial efficacy [36]. Also, there is a remarkable difference in molecular dimension between MB and TMPyP (319.85 g/mol and $1363.6 \mathrm{~g} / \mathrm{mol}$ without counterions, respectively). The extent of penetration of MB through the bacterial membrane should be easier compared to TMPyP. However, due to the thicker cell wall of Gram-positive $S$. aureus, penetration of MB might be more difficult compared to penetration through Gram-negative cell membrane of E. coli. Gollmer et al. showed that MB only enters the cell following cell wall damage. Accumulation of $\mathrm{MB}$ in the cytosol or cell wall during incubation in the dark was minor [42]. Also, for TMPyP, no intracellular uptake was shown in E. coli [23]. The lower accumulation of MB compared to TMPyP in $S$. aureus could also be explained by the expression of multidrug efflux pumps. Tegos et al. showed that inhibition of specific efflux pumps in $S$. aureus 

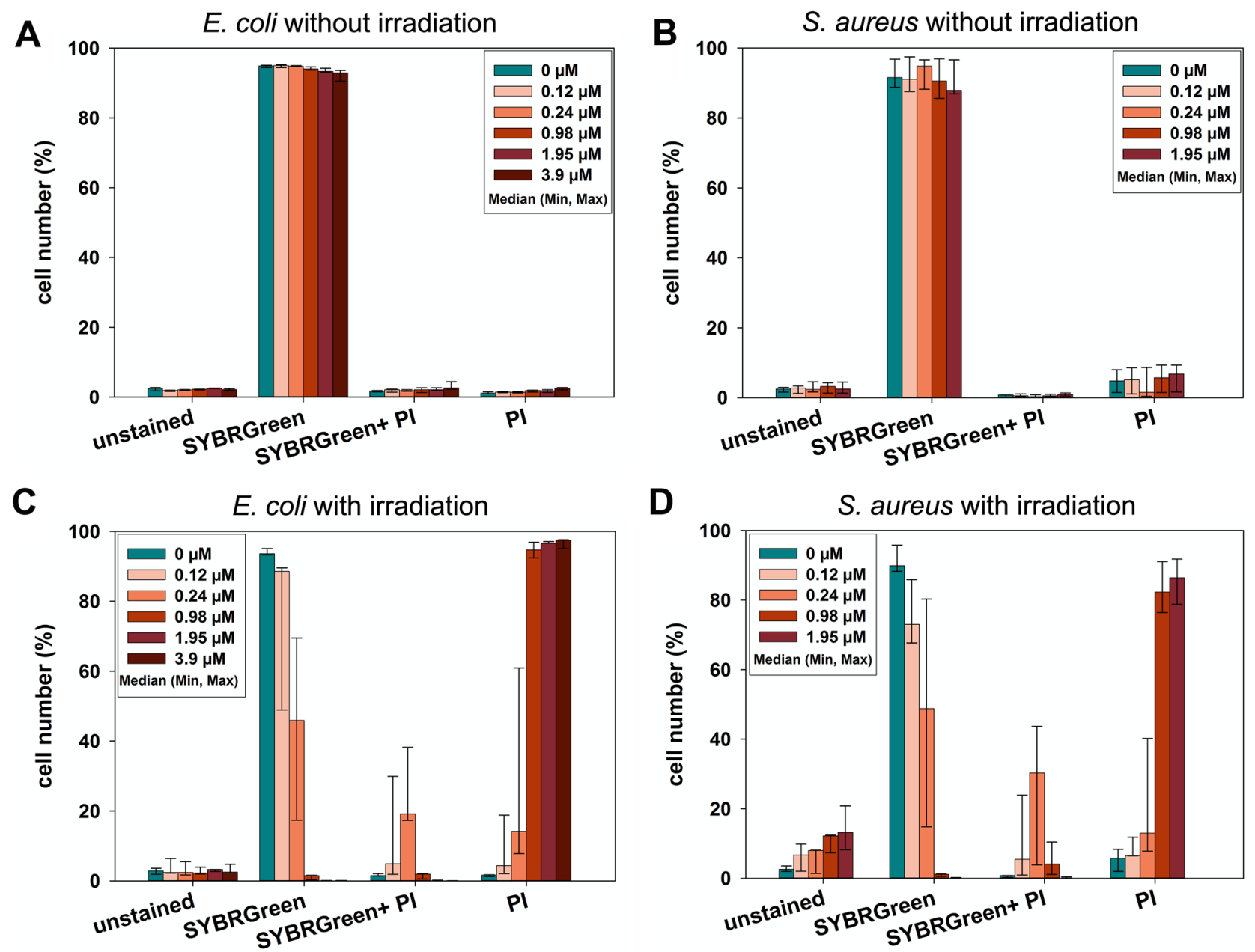

Fig. 7 Flow cytometry data on membrane integrity upon treatment of $E$. coli and $S$. aureus with TMPyP. Bacterial cell number in percent for unstained, SG-stained, SG + PI-stained, or PI-stained cells are shown. Values are presented as median, minimum, and maximum. A Values in percent for E. coli upon treatment with TMPyP

led to an increase in photodynamic killing using $\mathrm{MB}$ and other phenothiazinium dyes [43, 44].

Due to the high attachment of both PS tested to the bacteria, we assumed that the main target of the photodynamic process might be the bacterial cytoplasmic membrane. To investigate possible membrane damage, we performed flow cytometric analysis using SG and PI as described earlier $[10,30,45]$. The antiseptic CHX was chosen as a positive control because CHX is known for its underlying membranedamaging mechanism of action through the breakdown of the membrane potential $[46,47]$. The membrane-damaging effect of CHX on bacteria could be visualized in previous studies using flow cytometric analysis with PI [10, 30].

We found a membrane-damaging effect after photodynamic treatment with TMPyP and MB for E. coli and $S$. aureus. This result confirms the view in the literature that without irradiation; B Values in percent for $S$. aureus upon treatment with TMPyP without irradiation; C Values in percent for E. coli upon treatment with TMPyP with irradiation; D Values in percent for $S$. aureus upon treatment with TMPyP with irradiation; $n=3$

bacterial cytoplasmic membranes may be the primary target structure of the photodynamic mode of action [8,38, 48]. Furthermore, the present results are in accordance with other studies focusing on the membrane-damaging effect of photoinactivation on E. coli and S. aureus using MB and TMPyP [49-52]. For S. aureus, flow cytometric analysis showed that higher concentrations of MB were necessary to achieve membrane damage in most cells compared to $E$. coli. This could be due to the lower accumulation of MB by S. aureus. Also, the thicker cell wall structure might make Gram-positive bacteria less susceptible to membrane damage [53]. This is also in line with a recent study from our group. The flow cytometric analysis of biofilms formed by Gram-positive S. mutans and A. naeslundii or Gram-negative $E$. coli revealed a membrane-damaging effect on $E$. coli but not on both Gram-positive bacteria after treatment with aPDI 
using the phenalen-1-one derivative SAPYR as a PS [10]. However, there may be differences in the mode of action of PDI in biofilms compared to bacteria cultivated in planktonic cultures e.g., due to extracellular polymeric substances and a potentially reduced penetration depth throughout the biofilms [54, 55]. Furthermore, we found that in S. aureus, there was a significant increase of unstained cells following aPDI with MB and TMPyP. Both fluorescent dyes, SG I and PI, are known to intercalate with intact DNA [56]. Thus, the increase in unstained bacteria could be due to a breakage of DNA after aPDI treatment which may be caused by the formation of ROS. These findings are also consistent with those of our previous study in which aPDI using SAPYR led to an increase of unstained cells of $S$. mutans and $A$. naeslundii [10].

When comparing the CFU reductions with the flow cytometric results, we found there was only a small number of bacterial cells with clear membrane damage as shown by PI staining ( $10.7 \%$ for $S$. aureus at $3.9 \mu \mathrm{M} \mathrm{MB})$ but a reduction in bacterial ability to replicate of more than $5 \log _{10}$ steps. Compared to this finding, the measured membrane damage by TMPyP is consistent with the reduction in bacterial ability to replicate. A reason for this finding could be the formation of extracellular ROS due to aPDI e.g., $\mathrm{H}_{2} \mathrm{O}_{2}$, which then diffuse through the bacterial membrane resulting in intracellular damage of structures (lipids, proteins or nucleic acids) [57]. Accordingly, we found for the PS SAPYR an increase in intracellular ROS but no membrane damage for $S$. mutans and A. naeslundii [10]. Further investigations e.g., measurement of formation of intracellular ROS or expression of other stress markers are necessary to find more about the exact mechanism of action of MB.

In conclusion, we were able to show a concentrationdependent accumulation of the PS TMPyP and MB for planktonic cultures of Gram-negative E. coli and Grampositive $S$. aureus in vitro, whereby a higher accumulation of TMPyP and less accumulation of MB was found for $S$. aureus as compared to E. coli. Furthermore, we demonstrated a membrane-damaging effect in $S$. aureus and E. coli using both PS. We found that the reduction of the ability to replicate occurs with lower concentrations of MB than needed for membrane damage suggesting that membrane damage is not the only mechanism of aPDI using MB. In comparison, TMPyP showed an increase in membranedamaged cells at low concentrations suggesting that the cell membrane is the main target in aPDI using TMPyP. To further analyze possible targets of antimicrobial photodynamic inactivation, more information about the uptake or accumulation of PS by bacteria are needed. The oxidative damage of bacterial components depends on the localization of the photosensitizer to these structures as well as on the half-life and time-limited diffusion length of the resulting reactive oxygen species [8]. Therefore, for a future clinical application of aPDI systems, it is mandatory to investigate the mode of action not only for planktonic bacteria but also for biofilms.

Electronic supplementary material The online version of this article (https://doi.org/10.1007/s43630-021-00158-z) contains supplementary material, which is available to authorized users.

Author Contributions EB, TM, DG, FC, DM and KAH conceived and designed the experiments. EB performed the experiments. KAH, EB, DM, FC and TM analyzed the data. DM wrote the manuscript with input from all authors. All authors reviewed and approved the final version of the manuscript.

Funding Open Access funding enabled and organized by Projekt DEAL. This work was funded by the Deutsche Forschungsgemeinschaft (DFG, German Research Foundation; Grant CI 263/1-3).

Availability of data and material The original contributions generated for this study are included in the article/supplementary material, further inquiries can be directed to the corresponding author/s.

Code availability Not applicable.

\section{Declarations}

Conflict of interest The authors declare that they have no conflict of interest.

Ethical standards For this type of in vitro study, no ethical approval was required.

Consent to participate All authors confirm their participation of this manuscript.

Consent for publication All authors agree to publication.

Open Access This article is licensed under a Creative Commons Attribution 4.0 International License, which permits use, sharing, adaptation, distribution and reproduction in any medium or format, as long as you give appropriate credit to the original author(s) and the source, provide a link to the Creative Commons licence, and indicate if changes were made. The images or other third party material in this article are included in the article's Creative Commons licence, unless indicated otherwise in a credit line to the material. If material is not included in the article's Creative Commons licence and your intended use is not permitted by statutory regulation or exceeds the permitted use, you will need to obtain permission directly from the copyright holder. To view a copy of this licence, visit http://creativecommons.org/licenses/by/4.0/.

\section{References}

1. Rossolini, G. M., \& Mantengoli, E. (2008). Antimicrobial resistance in Europe and its potential impact on empirical therapy. Clinical Microbiology and Infection, 14, 2-8.

2. Harkins, C. P., et al. (2017). Methicillin-resistant Staphylococcus aureus emerged long before the introduction of methicillin into clinical practice. Genome biology, 18(1), 130-130. 
3. Cieplik, F., et al. (2019). Resistance toward chlorhexidine in oral bacteria-Is there cause for concern? Frontiers in microbiology, $10,587-587$.

4. Schwarz, S. R., et al. (2020). Limited antimicrobial efficacy of oral care antiseptics in microcosm biofilms and phenotypic adaptation of bacteria upon repeated exposure. Clinical Oral Investigation, 25(5), 2939-2950.

5. Nakonieczna, J., et al. (2019). Photoinactivation of ESKAPE pathogens: Overview of novel therapeutic strategy. Future Medicinal Chemistry, 11(5), 443-461.

6. Pendleton, J. N., Gorman, S. P., \& Gilmore, B. F. (2013). Clinical relevance of the ESKAPE pathogens. Expert Review of AntiInfective Therapy, 11(3), 297-308.

7. O'Neill, J., Tackling drug-resistant infections globally:final report and recommendations. Ro A, ed. Resistance, 2016. 84.

8. Cieplik, F., et al. (2018). Antimicrobial photodynamic therapywhat we know and what we don't. Critical Reviews in Microbiology, 44(5), 571-589.

9. Wainwright, M., et al. (2017). Photoantimicrobials-are we afraid of the light? The Lancet Infectious Diseases, 17(2), e49-e55.

10. Muehler, D., et al. (2020). Insights into mechanisms of antimicrobial photodynamic action toward biofilms using phenalen1-one derivatives as photosensitizers. Frontiers in Microbiology. https://doi.org/10.3389/fmicb.2020.589364

11. Cieplik, F., et al. (2018). Phenalen-1-one-mediated antimicrobial photodynamic therapy and chlorhexidine applied to a novel caries biofilm model. Caries Research, 52(6), 447-453.

12. Maisch, T., et al. (2007). Determination of the antibacterial efficacy of a new porphyrin-based photosensitizer against MRSA ex vivo. Photochemical \&amp; Photobiological Sciences, 6(5), 545-551.

13. Maisch, T., et al. (2007). The role of singlet oxygen and oxygen concentration in photodynamic inactivation of bacteria. Proceedings of the National Academy of Sciences USA, 104(17), 7223-7228.

14. Baptista, M. S., et al. (2017). Type I and type II photosensitized oxidation reactions: Guidelines and mechanistic pathways. Photochemistry and Photobiology, 93(4), 912-919.

15. de Oliveira, B. P., Aguiar, C. M., \& Câmara, A. C. (2014). Photodynamic therapy in combating the causative microorganisms from endodontic infections. European Journal of Dentistry, $8(3), 424-430$.

16. Foschi, F., et al. (2007). Photodynamic inactivation of Enterococcus faecalis in dental root canals in vitro. Lasers in Surgery and Medicine, 39(10), 782-787.

17. Garcez, A. S., \& Hamblin, M. R. (2017). Methylene blue and hydrogen peroxide for photodynamic inactivation in root canal-a new protocol for use in endodontics. European Endodontic Journal, 2(1), 29.

18. Wilkinson, F., Helman, W. P., \& Ross, A. B. (1993). Quantum yields for the photosensitized formation of the lowest electronically excited singlet state of molecular oxygen in solution. Journal of Physical and Chemical Reference Data, 22(1), 113-262.

19. João, P., et al. (2005). Methylene blue in photodynamic therapy: From basic mechanisms to clinical applications. Photodiagnosis and Photodynamic Therapy, 2(3), 175-191.

20. Hasegawa, G. K. F., et al. (2015). Photodynamic inactivation of Escherichia coli by methylene blue and malachite green under red LED light. Revista Ambiente \&amp; Água, 10, 318-326.

21. Sabbahi, S., et al. (2008). The role of reactive oxygen species in Staphylococcus aureus photoinactivation by methylene blue. Water Science and Technology, 58(5), 1047-1054.

22. Teichert, M. C., et al. (2002). Treatment of oral candidiasis with methylene blue-mediated photodynamic therapy in an immunodeficient murine model. Oral Surgery, Oral Medicine, Oral Pathology, Oral Radiology and Endodontics, 93(2), 155-160.
23. Preuss, A., et al. (2013). Photoinactivation of Escherichia coli (SURE2) without intracellular uptake of the photosensitizer. Journal of Applied Microbiology, 114(1), 36-43.

24. Hanakova, A., et al. (2014). The application of antimicrobial photodynamic therapy on $S$. aureus and E. coli using porphyrin photosensitizers bound to cyclodextrin. Microbiological Research, 169(2-3), 163-170.

25. Maisch, T., et al. (2012). Fast and effective: Intense pulse light photodynamic inactivation of bacteria. Journal of Industrial Microbiology and Biotechnology, 39(7), 1013-1021.

26. Eichner, A., et al. (2013). Dirty hands: Photodynamic killing of human pathogens like EHEC, MRSA and Candida within seconds. Photochemical \&amp; Photobiological Sciences, 12(1), 135-147.

27. Miles, A. A., Misra, S. S., \& Irwin, J. O. (1938). The estimation of the bactericidal power of the blood. Journal of Hygiene (London), 38(6), 732-749.

28. Pearson, R. D., et al. (1980). Method of reliable determination of minimal lethal antibiotic concentrations. Antimicrobial Agents and Chemotherapy, 18(5), 699-708.

29. Bloomfield, S. F., et al. (1991). Comparative testing of disinfectant and antiseptic products using proposed European suspension testing methods. Letters in Applied Microbiology, 13(5), 233-237.

30. Cieplik, F., et al. (2018). Phenalen-1-one-mediated antimicrobial photodynamic therapy: Antimicrobial efficacy in a periodontal biofilm model and flow cytometric evaluation of cytoplasmic membrane damage. Frontiers in Microbiology, 9, 688.

31. Nikaido, H. (2003). Molecular basis of bacterial outer membrane permeability revisited. Microbiology and molecular biology reviews : $M M B R, 67(4), 593-656$.

32. Spagnul, C., Turner, L. C., \& Boyle, R. W. (2015). Immobilized photosensitizers for antimicrobial applications. Journal of Photochemistry and Photobiology B: Biology, 150, 11-30.

33. Ergaieg, K., \& Seux, R. (2009). A comparative study of the photoinactivation of bacteria by meso-substituted cationic porphyrin, rose Bengal and methylene blue. Desalination, 246(1), 353-362.

34. Ghorbani, J., et al. (2018). Photosensitizers in antibacterial photodynamic therapy: An overview. Laser Therapy, 27(4), 293-302.

35. Zolfaghari, P. S., et al. (2009). In vivo killing of Staphylococcus aureus using a light-activated antimicrobial agent. BMC Microbiology, 9, 27-27.

36. Eckl, D., Huber, H., \& Bäumler, W. (2020). First report on photodynamic inactivation of archaea including a novel method for high-throughput reduction measurement. Photochemistry and Photobiology, 96(4), 883-889.

37. Cieplik, F., et al. (2015). The impact of absorbed photons on antimicrobial photodynamic efficacy. Frontiers in Microbiology, 6, 706.

38. Alves, E., et al. (2014). An insight on bacterial cellular targets of photodynamic inactivation. Future Medicinal Chemistry, 6(2), 141-164.

39. Parasuraman, P., et al. (2019). Synthesis and antimicrobial photodynamic effect of methylene blue conjugated carbon nanotubes on E. coli and S. aureus. Photochemical \&amp; Photobiological Sciences, 18(2), 563-576.

40. Faraj Tabrizi, P., et al. (2018). Susceptibility of sodA- and sodBdeficient Escherichia coli mutant towards antimicrobial photodynamic inactivation via the type I-mechanism of action. Photochemical \&amp; Photobiological Sciences, 17(3), 352-362.

41. Sonohara, R., et al. (1995). Difference in surface properties between Escherichia coli and Staphylococcus aureus as revealed by electrophoretic mobility measurement. Biophysical Chemistry, 55, 273-277. 
42. Gollmer, A., et al. (2017). Real-time imaging of photodynamic action in bacteria. Journal of Biophotonics, 10(2), 264-270.

43. Tegos, G. P., et al. (2008). Inhibitors of bacterial multidrug efflux pumps potentiate antimicrobial photoinactivation. Antimicrobial Agents and Chemotherapy, 52(9), 3202-3209.

44. Tegos, G. P., \& Hamblin, M. R. (2006). Phenothiazinium antimicrobial photosensitizers are substrates of bacterial multidrug resistance pumps. Antimicrobial Agents and Chemotherapy, 50(1), 196-203.

45. Cieplik, F., et al. (2019). Antimicrobial efficacy of alternative compounds for use in oral care toward biofilms from caries-associated bacteria in vitro. MicrobiologyOpen, 8(4), e00695.

46. Cheung, H.-Y., et al. (2012). Differential actions of chlorhexidine on the cell wall of Bacillus subtilis and Escherichia coli. PLoS One, 7(5), e36659-e36659.

47. Kuyyakanond, T., \& Quesnel, L. B. (1992). The mechanism of action of chlorhexidine. FEMS Microbiology Letters, 100(1-3), 211-215.

48. Maisch, T., et al. (2004). Antibacterial photodynamic therapy in dermatology. Photochemical \&amp; Photobiological Sciences, 3(10), 907-917.

49. Ehrenberg, B., et al. (1993). Electric depolarization of photosensitized cells: lipid vs. protein alterations. Biochimica et Biophysica Acta (BBA)- Biomembranes, 1151(2), 257-264.

50. Malik, Z., et al. (1993). Collapse of $\mathrm{K}+$ and ionic balance during photodynamic inactivation of leukemic cells, erythrocytes and Staphylococcus aureus. International Journal of Biochemistry, 25(10), 1399-1406.
51. Salmon-Divon, M., Nitzan, Y., \& Malik, Z. (2004). Mechanistic aspects of Escherichia coli photodynamic inactivation by cationic tetra-meso( $N$-methylpyridyl)porphine. Photochemical \&amp; Photobiological Sciences, 3(5), 423-429.

52. Müller, A., Preuß, A., \& Röder, B. (2018). Photodynamic inactivation of Escherichia coli-Correlation of singlet oxygen kinetics and phototoxicity. Journal of Photochemistry and Photobiology B: Biology, 178, 219-227.

53. Yin, R., et al. (2015). Antimicrobial photodynamic inactivation in nanomedicine: Small light strides against bad bugs. Nanomedicine (London, England), 10(15), 2379-2404.

54. Dunne, W. M., Jr., Mason, E. O., Jr., \& Kaplan, S. L. (1993). Diffusion of rifampin and vancomycin through a Staphylococcus epidermidis biofilm. Antimicrobial Agents and Chemotherapy, 37(12), 2522-2526.

55. Singh, R., et al. (2010). Penetration of antibiotics through Staphylococcus aureus and Staphylococcus epidermidis biofilms. Journal of Antimicrobial Chemotherapy, 65(9), 1955-1958.

56. Joux, F., \& Lebaron, P. (2000). Use of fluorescent probes to assess physiological functions of bacteriaat single-cell level. Microbes and Infection, 2(12), 1523-1535.

57. Vatansever, F., et al. (2013). Antimicrobial strategies centered around reactive oxygen species-bactericidal antibiotics, photodynamic therapy, and beyond. FEMS Microbiology Reviews, 37(6), 955-989. 